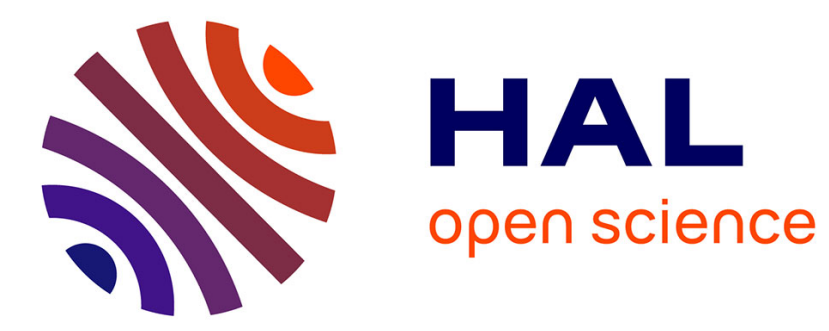

\title{
Self-Regulated Smectic Emulsion with Switchable Lasing Application
}

Dae Seok Kim, Wonsuk Lee, T. Lopez-Leon, Dong Ki Yoon

\section{To cite this version:}

Dae Seok Kim, Wonsuk Lee, T. Lopez-Leon, Dong Ki Yoon. Self-Regulated Smectic Emulsion with Switchable Lasing Application. Small, 2019, 15 (49), pp.1903818. 10.1002/smll.201903818 . hal02408506

\section{HAL Id: hal-02408506 https://hal.science/hal-02408506}

Submitted on 11 Jan 2021

HAL is a multi-disciplinary open access archive for the deposit and dissemination of scientific research documents, whether they are published or not. The documents may come from teaching and research institutions in France or abroad, or from public or private research centers.
L'archive ouverte pluridisciplinaire HAL, est destinée au dépôt et à la diffusion de documents scientifiques de niveau recherche, publiés ou non, émanant des établissements d'enseignement et de recherche français ou étrangers, des laboratoires publics ou privés. 


\title{
WILEY-VCH
}

\section{Self-regulated smectic emulsion with switchable lasing application}

Dae Seok Kim, Wonsuk Lee, Teresa Lopez-Leon* and Dong Ki Yoon*

Dr. D. S. Kim, Dr. T. Lopez-Leon

UMR Gulliver 7083 CNRS, ESPCI Paris, PSL Research University, 10 rue Vauquelin, 75005

Paris, France

E-mail: Teresa.Lopez-Leon@espci.fr

Dr. W. Lee

Nanophotonics Research Center, Korea Institute of Science and Technology, Seoul 02792,

South Korea

Prof. D. K. Yoon

Graduate School of Nanoscience and Technology, KAIST, Department of Chemistry and KINC, KAIST, Daejeon 34141, Republic of Korea

E-mail: nandk@kaist.ac.kr

Keywords: emulsion, liquid crystal, self-regulation, switchable lasing

\begin{abstract}
We present a structurally reversible smectic liquid crystal (LC) emulsion made of semifluorinated rod-type molecules in silicon oil, which is controlled by simple heating and cooling. Without adding any kind of additives, such as surfactants, polymers or emulsifiers, and without using any special tools, such as microfluidics or gas bubbling, the LC molecules spontaneously form monodisperse spherical and myelin-like structures upon cooling from the isotropic temperature. The LC emulsion can easily trap guest materials, providing a platform for repeatable and reliable switchable emulsification. For example, this interesting system enables to realize an on-off lasing system by confining fluorescent dyes in the LC droplets.
\end{abstract}




\section{WILEY-VCH}

Emulsions, in which droplets of a liquid are dispersed in an immiscible continuous phase, are widely used in commercial products including milk, paint, cosmetic products and medical treatment. A great amount of attention has recently been paid to switchable emulsions, which enable to realize a rapid transition between the emulsion state and the completely separated state. Such switchable emulsions have potential applications in colloidal science and engineering, for clearance of chemicals, drug delivery, ${ }^{[1,2]}$ switchable optical sensing, ${ }^{[3,4]}$ designing on-off opto-electronic devices, ${ }^{[5]}$ biocatalyst, ${ }^{[6]}$ or colloidal shape control. ${ }^{[7]}$ So far, switchable emulsions have been designed using oil-in-water or water-in-oil emulsions which are stabilized by switchable emulsifiers, ${ }^{[8,9]}$ which activate or deactivate upon the action of specific external stimuli such as $\mathrm{pH},{ }^{[10,11]}$ gas bubbling, ${ }^{[12]}$ light, ${ }^{[6]}$ or magnetic field. ${ }^{[13]}$ However, the use of water-in-oil emulsions enforces the switching mechanism to be a twostep process, since the separated liquids need specific mechanical treatments such as sonication or gas bubbling to return to the initial emulsion form, ${ }^{[8,9]}$ restricting the practical applications. Moreover, the switchable emulsifiers need to be specially designed according to the physical and chemical properties of the host/guest liquids and switching triggers, which makes this method complicated, time consuming, and expensive. Finally, although it has been demonstrated that self-emulsification methods can produce emulsions with small droplet size, i.e. submicron, without any mechanical energy input, these processes typically require high concentration of surfactants and are not reversible. ${ }^{[14-16]}$

An ideal switchable emulsion should fulfill several requirements. First, the guest liquid should be able to be emulsified in the host medium without the need of a specific emulsifier, since the presence of surfactants would compromise the purity of the involved liquids and potentially decrease the performance of the system in its active form. Second, for high reproducibility and reliability, the system should be capable of reversibly producing droplets of uniform size so that every single droplet in the emulsion performs its particular function in the same way. Third, the system should be able to undergo rapid and infinitely reversible on- 


\section{WILEY-VCH}

off switching without any mechanical treatment. In order to realize this, the host and guest liquids would need to be semi-miscible with a degree of miscibility that could be modulated through physical parameters, such as temperature of the guest material. Since semi-miscibility requires a mild hydrophobic interface between the two constituents, non-aqueous systems such as oil-in-oil emulsions seem to be appropriate candidates. Yet, oil-in-oil emulsions are usually unstable due to the low surface tension at the oil-oil interface, for instance, $\sim 3 \mathrm{mN} / \mathrm{m}$ for hydrocarbon and silicon oils. ${ }^{[17]}$ Stabilizing oil-in-oil emulsions without the use a specifically designed stabilizer is still a challenge. ${ }^{[18]}$

In this paper, we propose a method to fabricate highly stable switchable emulsions that fulfill the requirements mentioned above. The method consists in mixing silicon oil with a semifluorinated liquid crystal (LC) that display two different smectic phases (SmA and SmE) when decreasing temperature from the isotropic phase. We show that emulsification and demulsification processes occur at the smectic A and isotropic phases respectively, enabling fast switching between the two states via temperature. The elasticity and chemistry of the LC guarantees the stability of the emulsified state without the use of any surfactant. Interestingly, we observe two different kinds of morphologies in the emulsified state, spherical droplets and myelin-like filaments, resulting from an interesting interplay between kinetics of nucleation and growth, elasticity of the liquid crystal, and surface tension. Although these liquid crystalline organizations have been reported before, ${ }^{[19-21]}$ previous studies only focused on the formation and growth mechanisms of the structures, usually under confined geometries, while little effort has been paid to the realization of a switchable system where these structures could form and disappear in a completely reversible way, providing a solid platform for reconfigurable emulsification. In this paper, we show that the growth of filaments from a state where the oil and LC are fully mixed yields the formation of rather monodisperse emulsions, capable to switch between the emulsion and homogenous mixed state by thermal phase 


\section{WILEY-VCH}

transition. Finally, we show the potential of this system in terms of applications, for instance, as a switchable laser.

In order to make the oil-in-oil emulsion, we synthesized a semi-fluorinated LC molecule, Y002, whose structure is shown in Figure 1a. ${ }^{[22,23]}$ The Y002 was used as guest oil, while silicone oil was used as host, continuous phase. To prepare the emulsion sample, $0.1 \mathrm{wt} \%$ of the powdered Y002 is dispersed in $1 \mathrm{ml}$ of silicon oil and vigorously mixed for $2 \mathrm{~min}$. Then, the mixture is placed on a heating stage and heated up to the isotropic temperature, $200^{\circ} \mathrm{C}$, and cooled down at a rate of $10^{\circ} \mathrm{C} / \mathrm{min}$ to room temperature. A drop of this mixture is confined between a glass slide and a cover slip using $\sim 300 \mu \mathrm{m}$ spacers, and observed utilizing polarized optical microscopy (POM) with varying temperature. On cooling from the isotropic to room temperature, the Y002 adopts three different morphologies as shown in Figure 2 and Video S1: (i) a completely mixed state, when Y002 is in the isotropic phase (above $200^{\circ} \mathrm{C}$ ), see Figure 2a,d, (ii) a state where myelin-like smectic filaments are dispersed in silicon oil, which forms at the onset of the isotropic-smectic phase transition (at $185^{\circ} \mathrm{C}$ when cooling from the isotropic phase), see Figure $2 \mathrm{~b}$,e and eventually (iii) a state where onion-like smectic droplets are dispersed in silicon oil, which appears all over the temperature range of the smectic phase, see Figure 2c,f.

The emulsion state turned out to be extremely stable. This stability comes from two main factors. First, the semi-fluorinated functional group of the Y002 molecule acts as pseudoemulsifier: it places itself at the interface between silicon oil and Y002, lowering its surface tension. ${ }^{[24]}$ Thus, the semi-fluorinated group in Y002 plays the role of both guest oil and surfactant. The second and probably stronger stabilizing factor comes from the change in elastic properties of $\mathrm{Y} 002$ as the system is cooled down from the isotropic phase to the smectic A, smectic E and soft crystal phases. Cooling down to room temperature very quickly makes each droplet enter the soft crystal phase, in which molecular translation and rotation 


\section{WILEY-VCH}

are thermodynamically not allowed, and where the droplets exist as they were solid particles dispersed in the oil.

Once the emulsion is formed, it is possible to make the smectic droplets appear and disappear through thermal phase transition. We tested the switchable behavior of our emulsion platform in terms of switching speed, size uniformity of the droplets and reproducibility. The switching between the mixed and emulsified states occurs in a time interval of less than a second. The process is fully reversible and could be, in principle, indefinitely repeated (Figure 3a). Interestingly, the LC spherical droplets formed in the emulsification process are rather monodisperse, with a diameter of $\sim 13 \mu \mathrm{m}$ (Figure $3 \mathrm{a}$ ). Without using any specific tool such as microfluidic set-up, homogenizer, or ultrasonicator, our system produces uniformly sized spheres by temperature-induced self-assembly of the LC molecules. This is possible due to a specific mechanism of nucleation and growth, combined with the elastic properties of the smectic phase, as discussed later.

To get some quantitative information about the self-assembly and dispersion of the Y002 molecules during the emulsification and demulsification processes, we incorporated a red dye that is fully miscible with Y002, but poorly miscible with silicon oil. During repeated heating and cooling cycles, the color of the host medium, here silicon oil, becomes reddish at the dispersed state $\left(200^{\circ} \mathrm{C}\right)$ and whitens at the emulsified state $\left(120^{\circ} \mathrm{C}\right)$, indicating that the Y002 molecules and the dye get mixed with the silicon oil when Y002 is the isotropic phase $\left(200^{\circ} \mathrm{C}\right)$. The dispersed phase, i. e. the spheres and filaments, always exhibits an intense red color, meaning that the emulsion can trap red dyes when it is generated from a fully mixed state (Figure 3c and Video S2). To get some quantitative information on the mixing process, the red-color intensity variation in the silicon oil is measured as a function of temperature (Figure 3b). The intensity gradually increases upon heating to the isotropic phase $\left(200^{\circ} \mathrm{C}\right)$, meaning that the LC molecules are progressively dispersed into the silicon oil, eventually reaching a homogeneous state where both Y002 and silicon oil are fully mixed. When cooling 


\section{WILEY-VCH}

down to the smectic phase at a rate of $10^{\circ} \mathrm{C} / \mathrm{min}$, the intensity profile associated to the silicon oil gets reversed, but the intensity values become $\sim 8 \%$ larger than in the heating experiment, indicating that a small amount of red dye remains in the silicon oil after the phase transition. This color change of the host medium with temperature shows that the miscibility between Y002 and silicon oil is intrinsically poor, except when Y002 is in the isotropic phase (> $200^{\circ} \mathrm{C}$ ). Such a specific miscibility of the LC and silicon oil depending on the LC phase allows for rapid conversion between the emulsion and mixed states, facilitating the switching behavior of the emulsion.

The molecular structures associated to the different droplet morphologies are sketched in Figure 1b. In the SmA phase, the Y002 molecules are perpendicularly anchored to the interface with silicon oil, which prescribes homeotropic boundary conditions. ${ }^{[25]}$ When the liquid crystal is confined to a spherical volume, these boundary conditions typically result in an onion-like organization of the smectic layers, as represented in Figure 1b (i). This concentric configuration assures the compliance of both boundary conditions and equidistance between smectic layers. The Maltese cross texture appearing when placing the sample between cross polarizers, together with the blue and yellow colors arising when adding a wave retardation plate $(\lambda=530 \mathrm{~nm})$, confirms the concentric structure of the spherical droplets, as shown in the inset of Figure $2 \mathrm{c}$ and in Figure $\mathrm{S} 1$ in the supporting information). In their elongated shape, the Y002 droplets take the shape of spherocylinders, where two spherical caps are connected by a long cylinder. The molecular organization within the cylinder is shown in Figure 1b (iii), where the smectic layers are organized in a coaxial fashion.

Because of the curvature of the smectic layers, both the spherical and cylindrical structures hold topological defects, i. e. singular regions where the smectic order is ill-defined. A concentric structure of spherical smectic layers produces a point defect at the center of the structure, highlighted in red in Figure 1b (i). In contrast, a coaxial structure of cylindrical smectic layers leads to the formation of a defect line or disclination along the symmetry axis 


\section{WILEY-VCH}

of the structure, highlighted in red in Figure 1b (ii). As one approaches the center of the concentric/coaxial structure, curvature gradients and thus elastic energy density dramatically increase. In nematics, the cylindrical structure can release this elastic energy by letting the molecules escape into the third dimension at the core of the cylinder. However, smectics cannot escape, so they release energy by locally melting those regions that localize curvature. ${ }^{[26,27]}$ This is confirmed by the POM images, which reveal an extinction (dark) line along the axis of the filament (inset Figure 2b), indicating the existence of an isotropic phase at the core of the cylinder. This core-shell structure becomes even more evident when examining the sample through scanning electron microscopy (SEM), which allows for threedimensional visualization of the structure. In these measurements, the sphere and filament structures were quenched, collected from the silicon oil by filtering, and coated by a $5 \mathrm{~nm}$ platinum film to enhance image contrast. The resulting images are shown in Figure $2 \mathrm{f}$, for the spherical geometry, and Figure 2e for the cylindrical one. Although the surface of each structure does not show any remarkable texture, the cross section of the filament, obtained by freeze fracture transmittance electron microscopy (FF-TEM), shows two distinct phases: a disordered core of about $\sim 500 \mathrm{~nm}$ surrounded by a thick shell of concentric smectic layers (inset of Figure 2e).

The formation of both spherical droplets and smectic filaments results from an interesting process where kinetics of growth and elastic energy interplay. The filaments are a nonequilibrium transient forms raised by fast cooling from the isotropic phase. As previously reported, ${ }^{[28]}$ the formation of filaments may be preceded by the nucleation of two dimensional membranes of Y002 in the silicon oil, which are unstable and roll up into cylinders due to the anisotropic surface tension of the LC sheet. The elongation of the filaments are accompanied of buckling, which is driven by the internal force (load) produced by the growth of the smectic phase. ${ }^{[29]}$ The load decreases as the filaments increase in length, making bucking less important when the filaments become long. Although spherical nucleii are also formed when 


\section{WILEY-VCH}

temperature decreases below the critical one, the growth of smectic filaments is kinetically favored with respect to their spherical counterparts (Video S1). Indeed, isotropic growth is sustained by the local uptake of molecules, while longitudinal growth makes the structure travel through the sample, having fast access to molecules that are far from the initial nucleation spot. As in typical crystallization processes, the presence of a growing structure prevents the local formation of other ones by the uptake of neighboring molecules, this is why the formation of filaments is favored near the isotropic / smectic phase transition temperature (Video S1).

The filaments become unstable and transform into spherical droplets by fast cooling with a rate of $10^{\circ} \mathrm{C} / \mathrm{min}$. When decreasing $\mathrm{T}$, the $\mathrm{Y} 002$ molecular interactions become stronger with respect to $k_{B} T$, resulting in the increase of the elastic constants of the liquid crystal. ${ }^{[30,31]}$ In the smectic A phase, the elastic energy per unit volume is mainly controlled by the splay term, which takes into account the mean curvature of the smectic layers: ${ }^{[32]}$

$$
f_{e}=\frac{K}{2} H ; \quad H=\left(\frac{1}{R_{1}}+\frac{1}{R_{2}}\right)^{2}
$$

where $K$ is the splay elastic constant and $H$ is the mean curvature of the smectic layers $\left(R_{1}\right.$ and $R_{2}$ are principal curvature radii). In order to understand the transformation of the filaments into spheres, let consider a long filament and a sphere with the same volume: because of the larger mean curvature of the filament, the associated elastic energy is also larger, making the structure more energetically costly than the spherical one. ${ }^{[33]}$ Although the formation of filaments is kinetically favored, they become energetically unstable as elastic energy comes into play. Reaching the middle of the temperature range for the smectic phase (below $185^{\circ} \mathrm{C}$ ), all the filaments are transformed into spherical droplets. Looking closely at the contraction dynamics of the filament, it was frequently observed that two spheres start to be formed at both ends of the filament at the initial stages of the process and then, as the two spheres grow, the filament connecting them becomes progressively stretched, until it 


\section{WILEY-VCH}

eventually breaks (see video S3 and figure S2). Although it is extremely hard to measure a critical length or radius for the fracture of the filament, it seems obvious that the splitting of the filament is responsible for the rather uniform size of the spherical droplets obtained with this method. Upon further cooling to room temperature, the spheres adopt a smectic E phase and eventually a soft crystal phase. ${ }^{[34]}$ The LC spheres stop their growing when they become solidified, which limits the uptake of free molecules/aggregates and prevents Ostwald ripening in the solution. As a result, the size of the final LC spheres is rather uniform (Figure 3a).

In order to discuss the stability of the arrangement in concentric layers, we compare elastic energy, which tends to reduce the curvature of the layers, with anchoring energy, which forces the smectic layers to curve so that the Y002 molecules are perpendicular to the interface with the silicon oil. While the former scales as $W R^{2}$, the latter scales as $K R$, where $R$ is the radius of the droplet and $W$ is the anchoring coefficient. ${ }^{[27]}$ For large values of the radius, $R>K / W$, the anchoring energy penalty is significantly larger than the elastic cost of the concentric packing, favoring the concentric structure within the droplet. Here, it is extremely hard to measure the exact value of the surface anchoring energy between Y002 and the silicone oil at the isotropic-smectic phase transition because of the high temperature at which the transition takes place $\left(\sim 190^{\circ} \mathrm{C}\right)$. Yet, we can get an estimation of $W$ by measuring the smallest radius of the concentric smectic layers, $R_{\mathrm{S}}, \sim 1 \mu \mathrm{m}$, appearing when quenching the sample to room temperature from the isotropic-smectic phase transition temperature, where smectic layers starts forming (Figure S3). We assume the characteristic length $K / W$ is approximately equal to $R_{\mathrm{S}}$, thus, $W \approx K / R_{\mathrm{S}} \approx 3 \times 10^{-5} \mathrm{~N} / \mathrm{m}$, considering the average value of the Frank elastic constant $K \sim$ $30 \mathrm{pN} .^{[27,35]}$ Thus, for $W=3 \times 10^{-5} \mathrm{~N} / \mathrm{m}$ and the elastic constant $K=30 \mathrm{pN}$, the concentric layering with circular curvature should be valid as long as $R>K / W$, that is, practically for all scales exceeding $1 \mu \mathrm{m}$. Interestingly, the central defects observed in our concentric layered structures have anomaly large cores, $\sim 500 \mathrm{~nm}$, compared to those observed in conventional 


\section{WILEY-VCH}

thermotropic LCs, $\sim 10 \mathrm{~nm} .{ }^{[27]}$ Such large disordered defect cores have been frequently observed in spherical droplets of lyotropic LCs, where the liquid crystalline molecules are dispersed in a solvent. The origin of such large defect cores has been attributed to a larger concentration of solvent, which can even lead to a complete phase separation, at the defect core ${ }^{[36,37]}$ Since our system also has two different components, Y002 and silicon oil, it seems reasonable to make the assumption that the defect core may also contain silicon oil. To prove this hypothesis, we designed an experiment where a fluorescent dye (Coumarin 153, SigmaAldrich), soluble in silicon oil but not in the LC, was used to analyze the composition of the defect cores. The marked sample was quenched to room temperature from the isotropicsmectic phase transition temperature. As shown in the fluorescent confocal microscope images (Figure S4), the droplets contain silicon oil in their cores, as expected, explaining the larger size of these defects in comparison with standard (mono-component) thermotropic liquid crystals.

As the potential applications of our switching emulsion, we investigated the possibility of making a switchable laser. This application takes advantage of two properties of our system: the fast switching between the uniform and emulsified states and its capability of selectively trapping dyes inside the LC droplets. Figure 4a shows sequential fluorescent micrographs of LC droplets doped with Rhodamine $6 \mathrm{G}$ when varying temperature from $120^{\circ} \mathrm{C}$ to $200^{\circ} \mathrm{C}$, clearly showing thermal phase transition from smectic to isotropic and vice versa. The behavior is analogous to that is shown in Figure 3, although here the dye is more strongly associated with the LC molecules. Clear fluorescence is observed only inside the LC droplets at the smectic phase, while a uniform fluorescent background is observed at the isotropic phase, where the LC and dye molecules are fully dispersed through the entire silicon oil medium.

To test the lasing ability of our doped LC droplets, they were optically pumped with an external light source (5 ns pulsed laser, optical parametric oscillator, $532 \mathrm{~nm}$ ) through a 


\section{WILEY-VCH}

confocal setup, while a lasing signal was simultaneously collected by a spectrometer. The optical index of the silicon oil $(\mathrm{n} \sim 1.4)$ is lower than that of conventional calamitic smectic LC $\left(n_{0} \sim 1.516\right),{ }^{[38]}$ thus, we expect the Y002 droplets to support whispering gallery modes (WGMs) to confine light. Since the LC spheres are doped with an organic laser dye, Rhodamine 6G, each individual LC droplet provides gain for lasing activities, and at the same time, serve as a laser microcavity. Figure $4 \mathrm{~b}$ depicts a representative lasing spectrum for the smectic droplets at $120^{\circ} \mathrm{C}$, when the pump energy density is $3.1 \mathrm{~mJ} / \mathrm{mm}^{2}$. The lasing emission has multi-mode lasing peaks due to the small spectral range of the WGMs in the $\sim 13 \mu \mathrm{m}$ sized LC droplets, as well as coexistence of different TM and TE modes along the equator of the droplet. To further verify whether this is a lasing phenomenon, we measured the spectrally integrated optical emission intensities as a function of the pump energy density. Figure $4 \mathrm{c}$ shows that the fluorescence signal is virtually negligible before reaching a threshold, over which it gets abruptly amplified. This nonlinear response of the output signal is a direct proof of lasing. Above the threshold, the laser intensity linearly increases with the pump energy density. The lasing threshold of our LC spherical laser system, estimated from the linear fit of the curve, is $0.54 \mathrm{~mJ} / \mathrm{mm}^{2}$. This value is a few orders of magnitude higher than in other laser devices with similar R6G dye concentrations utilizing solid-state microcavities ${ }^{[39]}$ due to a lower Q-factor. That mainly refers to small refractive index contrast between Y002 and the surrounding medium (silicon oil) and the small size of the droplets. Yet, the LC spheres provide enough feedback for substantial lasing, leading to reliable laser demonstration with our system.

The Y002 molecules get dispersed into the silicon oil as temperature increases, and the entire medium exhibit fluorescence when externally pumped. Since light confinement by the LC sphere is absent, the medium simply shows typical fluorescence emission but not lasing, as indicated in Figure 4d, even though the system is pumped with same pump energy as in Figure $4 \mathrm{~b}$. Note that the nature of the R6G organic dye is not affected by the temperature 


\section{WILEY-VCH}

change. The lasing behavior of our switchable emulsion can be reproduced with other dyes, as long as the association between the LC and the guest dye is successful. For instance, using BTBP instead of R6G as a dopant leads to similar results, as illustrated in Figure S5. The only difference is the relatively small output emission and increased lasing threshold compared to the former case of R6G, due to the lower quantum efficiency of the BTBP dye. These results demonstrate that our LC/oil emulsion platform enables producing a large number of microlasers that can be switched on and off simply by modifying temperature.

In conclusion, we have designed a switchable oil-in-oil emulsion using LC and silicon oil as guest and host materials, respectively, which is operated by simple thermal stimuli. By taking advantage of fluorochemistry, the LC, Y002, was designed and used as both guest-oil and emulsifier. Due to the liquid crystallinity of Y002 and semi-miscibility between Y002 and silicon oil, the transition between emulsification and demulsification is reversibly triggered by varying temperature. Interestingly, the droplets adopt a transient filament-like structure upon fast cooling from the isotropic to the smectic phase, which plays a significant role in determining the final diameter of the LC droplets, leading to a emulsifiable system with uniform droplet size and repeatable and reliable switching properties. This switching behavior enabled us to successfully apply our emulsion to get on-off lasing performance by confining organic laser dyes in the smectic phase.

To our best knowledge, our system is the first example of switchable oil-in-oil emulsion that does not require any surfactant or emulsifier, fulfilling the conditions required for an ideal switchable emulsion. It also provides an interesting example of switchable opto-electric device based on emulsions. With future efforts to better control the shape of the droplets and the reflective index matching, we envisage that our tunable laser system may be useful for real applications, e.g., as a smart thermal sensor.

\section{Experimental Section}

\section{Materials}




\section{WILEY-VCH}

The LC material Y002 was synthesized by the procedure for the alkylation of methyl ethyl 4'hydroxy-4-biphenyl carboxylate with $1 \mathrm{H}, 1 \mathrm{H}, 2 \mathrm{H}, 2 \mathrm{H}, 3 \mathrm{H}, 3 \mathrm{H}, 4 \mathrm{H}, 4 \mathrm{H}$ perfluorododecyl bromide in DMF (Sigma Aldrich, $99.8 \%$ ) at $70{ }^{\circ} \mathrm{C}$ under reflux with $\mathrm{K}_{2} \mathrm{CO}_{3}{ }^{[22]}$

\section{Sample preparation}

$1 \mathrm{wt} \%$ of crystalline powdered Y002 is added to silicon oil and vigorously stirred at $200^{\circ} \mathrm{C}$ for $1 \mathrm{~min}$. The mixture is infiltrated into a cell where two glass substrates are separated by $100 \mu \mathrm{m}$ spacers made of polyimide tape $(3 \mathrm{M})$.

For the red dyed sample, $0.1 \mathrm{wt} \%$ of oil soluble red dye (PYLAM dyaes) was added to above emulsion mixture and vigorously stirred at $200^{\circ} \mathrm{C}$ for $1 \mathrm{~min}$. Then, the sample was diluted in pure silicon oil so that the red dye was kept only in the LC spheres (figure S6).

For the lasing test, the florescent LC spheres were prepared by adding $0.01 \mathrm{wt} \%$ of rhodamine 6G (Sigma Aldrich) or N,N-Bis(2,5-di-tert-butylphenyl)-3,4,9,10-perylenedicarboximide (BTBP, Sigma Aldrich) to the mixture by following the same procedure as with the red dye, and diluted so that the red dye was kept only in the LC spheres (figure S7).

To control the morphological changes of the LC droplets with temperature, the sample was heated and cooled with a rate of $10^{\circ} \mathrm{C} / \mathrm{min}$ on a heating stage (LINKAM LTS350) controlled by a temperature controller (LINKAM TMS94).

\section{Characterization}

The LC samples were directly imaged using polarized optical microscopy (POM) (LV100POL, Nikon) and field-emission SEM (FE-SEM, Hitachi, S-4800). Fluorescent images of the optical textures were obtained by fluorescence confocal microscopy (C2 plus, Nikon) with a $488 \mathrm{~nm}$ light source (Coherent). For cross sectional images of LC sample, the samples were prepared by using freeze fracture (BalTec BAF060) and observed by using Transmission Electron Microscopy (TEM, Tecnai G2 F20). 


\section{WILEY-VCH}

Supporting Information is available from the Wiley Online Library or from the author.

\section{Acknowledgements}

This work was supported by the National Research Foundation of Korea (NRF), grant funded by the Korean government (MSIT) 2017M3C1A3013923 and 2017R1E1A1A01072798, and the French National Research Agency (ANR), Grant No. 13-JS08-0006-01.

D.S.K. and D.K.Y. designed the research. D.S.K. and W.L. performed the experiments. D.S.K., W.L., T.L.L. and D.K.Y. analyzed experimental data and wrote the manuscript. D.S.K and W.L. are equally contributed to this work and D.K.Y. and T.L.L. are cocorresponding authors.

Received: ((will be filled in by the editorial staff))

Revised: ((will be filled in by the editorial staff)) Published online: ((will be filled in by the editorial staff))

\section{References}

[1] V. S. Baravkar, A. S. Jirage, V. K. Kate, S. A. Payghan, J. I. D’Souza, Asian j. biomed. pharm. sci. 2014, 4, 10.

[2] V. Jaitely, T. Sakthivel, G. Magee, A.T. Florence, J. Drug Del. Sci. Tech. 2004, 14, 113.

[3] I-H. Lin, D. S. Miller, P. J. Bertics, C. J. Murphy, J. J. de Pablo, N. L. Abbott, Science 2011, 332, 1297.

[4] Z. Xie, H. Ge, J. Du, T. Duan, G. Yang, Y. He, Anal. Chem., 2018, 90, 11665.

[5] A. Ghosh, T.K. Mallick, Renewable Energy 2018, 120, 126.

[6] Z. Chen, L. Zhou, W. Bing, Z. Zhang, Z. Li, J. Ren, X. Qu, J. Am. Chem. Soc. 2014, 136, 7498.

[7] N. Denkov, S. Tcholakova, I. Lesov, D. Cholakova and S. K. Smoukov, Nature, 2015, 528, 392.

[8] P. Liu, W. Lu, W.-J. Wang, B.-G. Li, S. Zhu, Langmuir 2014, 30, 10248.

[9] J. Tang, P. J. Quinlan, K. C. Tam, Soft Matter, 2015, 11, 3512.

[10] A. L. B. Maçon, S. U. Rehman, R. V. Bell, J. V. M. Weaver, Chem. Commun., 2016, 52, 136.

[11] J. V. M. Weaver, S. P. Rannard, A. I. Cooper, Angew. Chem. Int. Ed. 2009, 48, 2131.

[12] J. Jiang, Y. Zhu, Z. Cui, B. P. Binks, Angew. Chem. Int. Ed. 2013, 52, 12373. 


\section{WILEY-VCH}

[13] Y. Chen, Y. Bai, S. Chen, J. Ju, Y. Li, T. Wang, Q. Wang, ACS Appl. Mater. Interfaces 2014, 6, 13334.

[14] C. Solans, I. Sole', Curr. Opin. Colloid Interface Sci 2012, 17, 246.

[15] N. L. Sitnikova, R. Sprik,* G. Wegdam and E. Eiser, Langmuir, 2005, 21, 7083.

[16] A. Perazzo, V. Preziosi, S. Guido, Adv. Colloid Interface Sci. 2015, 222, 581.

[17] B. D. Hamlington, B. Steinhaus, J. J. Feng, D. Link, M. J. Shelley, A. Q. Shen, Liquid Crystals, 2007, 34, 861.

[18] B. P. Binks, A. T. Tyowua, Soft Matter, 2016, 12, 876.

[19] I. Sakurai, Y. Kawamura, Biochimica et Biophysiea Acta, 1984, 777, 347.

[20] V. Luzzati, A. Tardieu, Annu. Rev. Phys. Chem., 1974, 25, 79.

[21] M. Urbanski, C. G. Reyes, J. H. Noh, A. Sharma, Y. Geng, V. S. R. Jampani, J. P. F. Lagerwall, J. Phys.: Condens. Matter 2017, 29, 133003.

[22] D. K. Yoon, M. C. Choi, Y. H. Kim, M. W. Kim, O. D. Lavrentovich, H.-T. Jung, Nat. Mater. 2007, 6, 866.

[23] D. S. Kim, Y. J. Cha, M. H. Kim, O. D. Lavrentovich, D. K. Yoon, Nat. Comm. 2016, $7: 10236$.

[24] H. Hoerauf, K. Kobuch, J. Dresp, D.-H. Menz, Graefe's Arch Clin Exp Ophthalmol 2001, $239,373$.

[25] J. Jeong, M. W. Kim, Phys. Rev. Lett. 2012, 108, 207802.

[26] P. E. Cladis, M. Kleman, J. Phys. 1972, 33, 591.

[27] M. Kleman, O. D. Lavrentovich, Soft Matter Physics An Introduction. New York Springer, 2003.

[28] A. Adamczyk, Mol. Cryst. Liq. Cryst. 2000, 351, 145.

[29] M. Todorokihara, Y. Iwata, H. Naito, Phys. Rev. E, 2004, 70, 021701.

[30] L. Cheung, R. B. Meyer, H. Gruler, Phys. Rev. E, 1973, 31, 349.

[31] S. - D. Lee, R. B. Meyer, J. Chem. Phys, 1986, 84, 3443. 


\section{WILEY-VCH}

[32] Y. H. Kim, D. K. Yoon, M. C. Choi, H. S. Jeong, M. W. Kim, O. D. Lavrentovich, and H.-T. Jung, Langmuir 2009, 25, 1685

[33] G. Toquer, T. Phou, S. Monge, A. Grimaldi, M. Nobili, C. Blanc, J. Phys. Chem. B, 2008, $112,4157$.

[34] D. K. Yoon, Y. H. Kim, D. S. Kim, S. D. Oh, I. I. Smalyukh, N. A. Clark, H.-T. Jung, Proc. Natl. Acad. Sci. U S A, 2013, 110, 19263.

[35] K.-J. Lee, S.-J. Kim,1 D. Kang, J.-H. Kim, Opt. Exp. 2015, 23, 24903.

[36] Y.-K. Kim, S. V. Shiyanovskii, O. D. Lavrentovich, J. Phys.: Condens. Matter 2013, 25, 404202.

[37] Y. Li, J. J.-Y. Suen, E. Prince, E. M. Larin, A. Klinkova, H. T.-Aubin, S. Zhu, B. Yang, A. S. Helmy, O. D. Lavrentovich, E. Kumacheva, Nat. Commun. 2016, 7:12520.

[38] K. Peddireddy, V. S. R. Jampani, S. Thutupalli, S. Herminghaus, C. Bahr, and Igor Mǔ sev̌c, Opt. Express. 2013, 21, 198492.

[39] A. Palatnik, Y. R. Tischler, IEEE Photonic. Tech. L. 2016, 28, 1823. 


\section{WILEY-VCH}

(a)
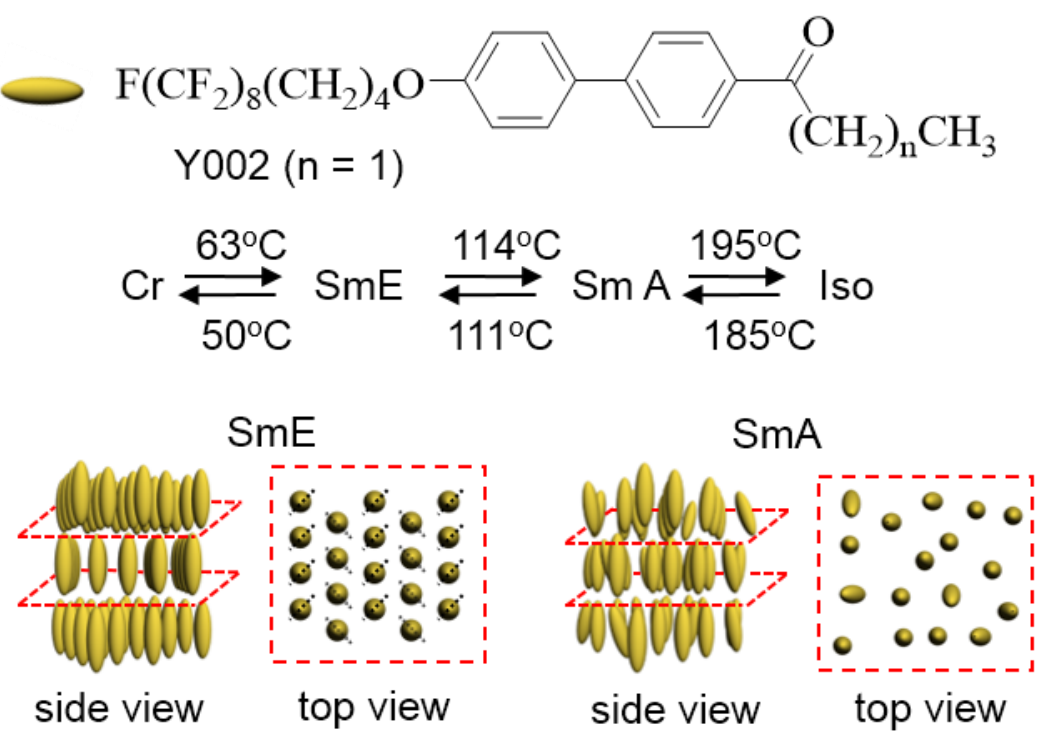

(b)
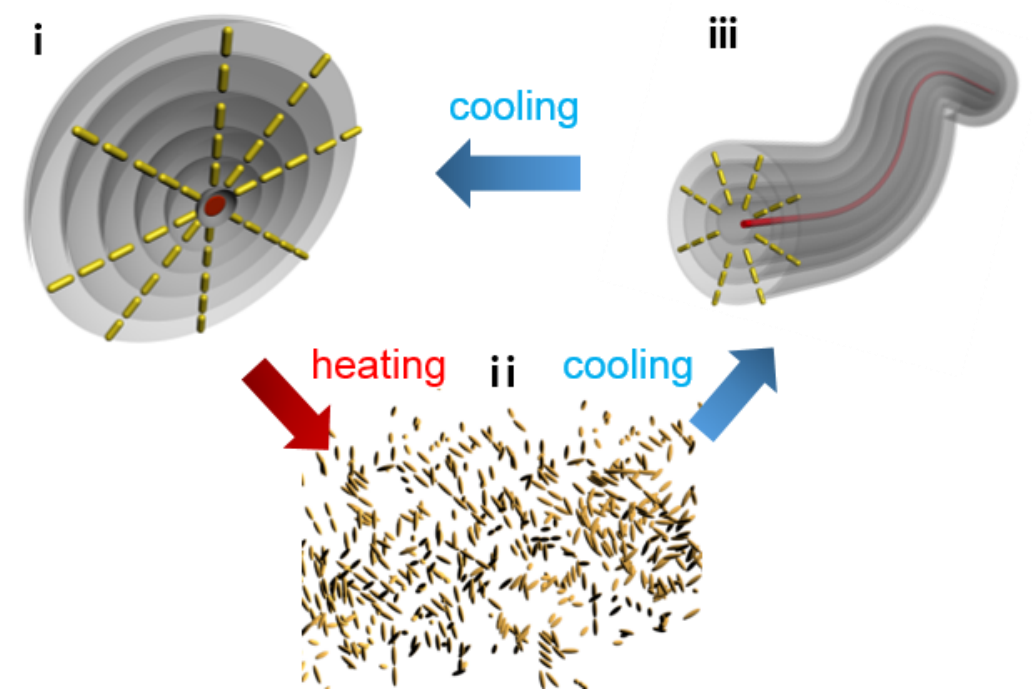

Figure 1. (a) Molecular structure and phase transition temperatures of Y002, where Cr, SmE, $\mathrm{SmA}$ and iso refer to the crystal, smectic E, smectic A and isotropic phase, respectively. The schematics show the molecular organization in the smectic A and smectic E phases, where the black dashed lines indicate plane of the biphenyl group of Y002. (b) Schematic illustration of the reversible emulsification process of the LC in the silicon oil medium induced by temperature. The fluorinated part of the LC molecule is oriented towards the silicon oil due to relatively lower surface tension it induces compared to the bi-phenyl part. The red dot and line in sphere and cylinder, respectively, indicate the presence of topological defects. 


\section{WILEY-VCH}
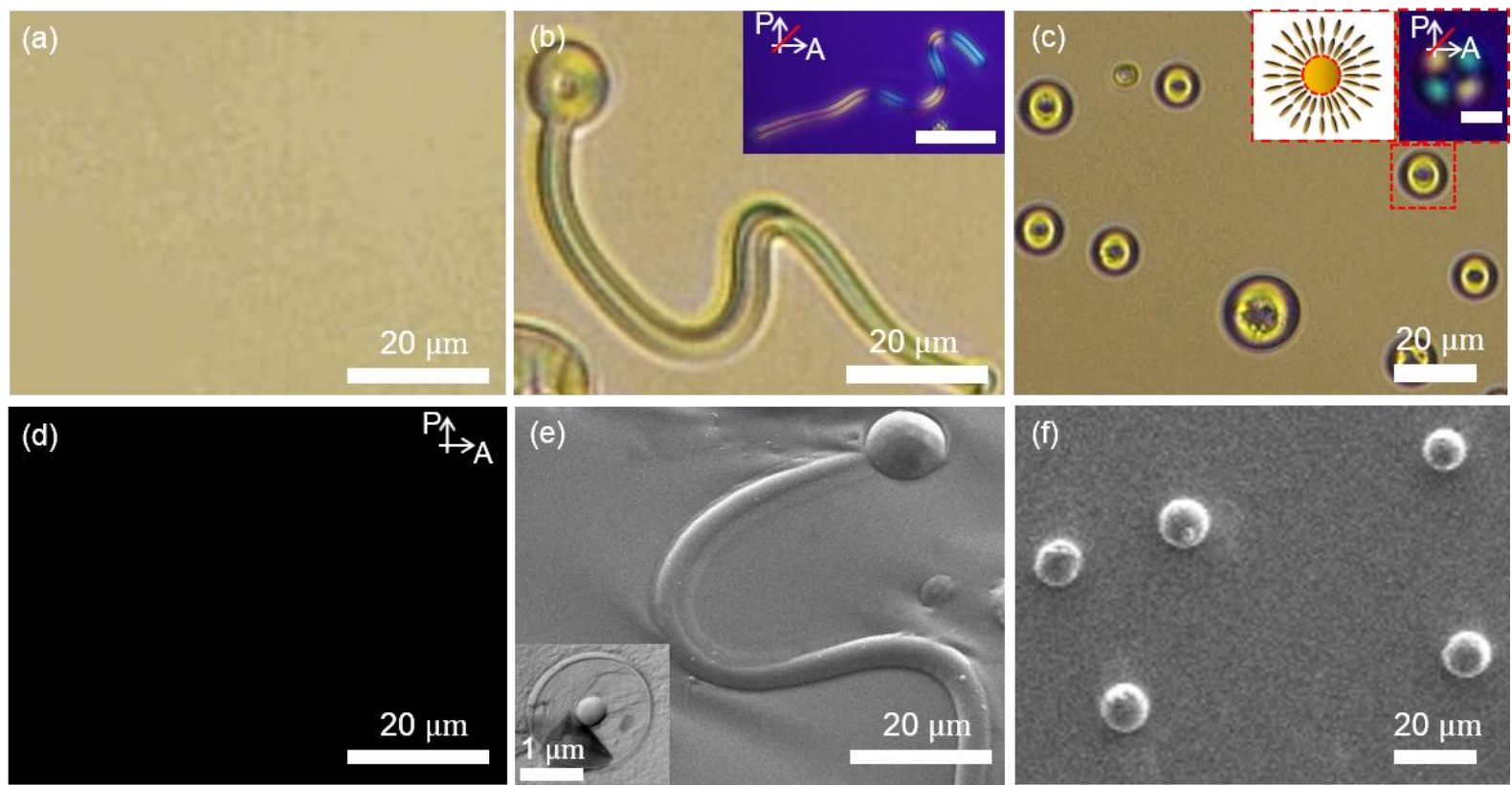

Figure 2. (a,d) $\mathrm{OM}$ and POM images of the well-mixed state at isotropic temperature, $200^{\circ} \mathrm{C}$, where the LC and silicon oil molecules form a continuous phase. (b) OM image of a filament structure dispersed in silicon oil; the inset shows the filament POM image with a retardation plate. (c) OM image of LC spheres dispersed in silicon oil; the insets show a POM image with a retardation plate (right) revealing the radial configuration of the droplet (left). (e,f) SEM images of a LC filament and spheres, respectively. The inset of (e) shows the cross section of a LC filament obtained by FF-TEM.

(a)

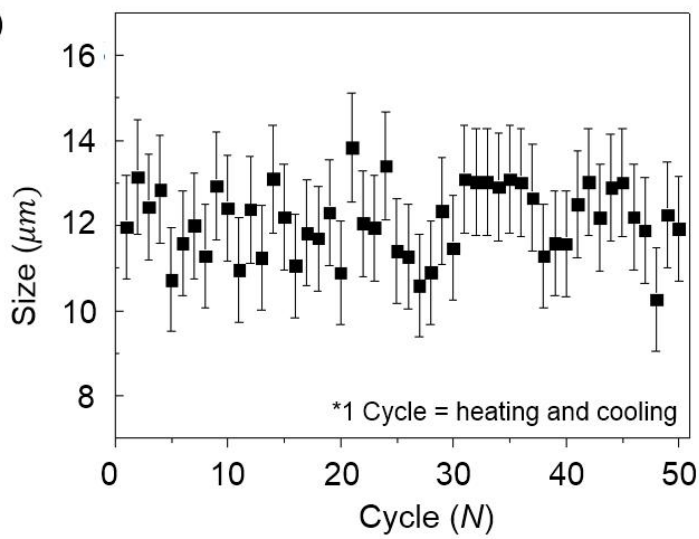

(b)

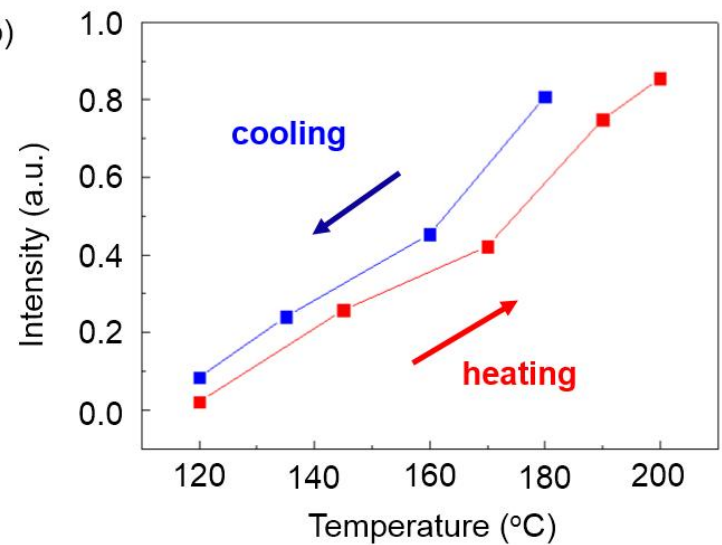

(c)
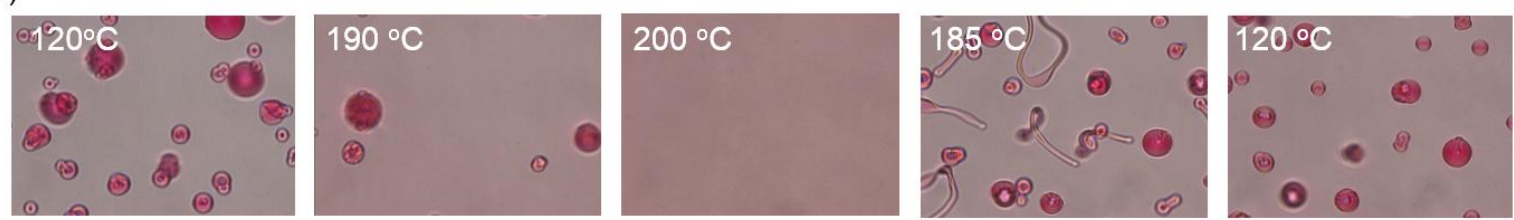

Figure 3. (a) Average size of LC spheres as a function of the number of transition cycles from isotropic $\left(200^{\circ} \mathrm{C}\right)$ to smectic phase $\left(120^{\circ} \mathrm{C}\right)$ (the number of spheres measured in each cycle is around 100) (b) Intensity profiles of the silicon oil during heating and cooling process when the LC is doped with red dye molecules, showing homogeneous dispersion of LC molecules upon demulsification in the isotropic phase (c) Sequential OM images of the reversible emulsification of the LC by thermal phase transition from smectic to isotropic phase. 


\section{WILEY-VCH}

(a)

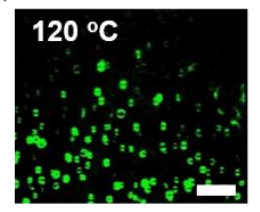

(b)

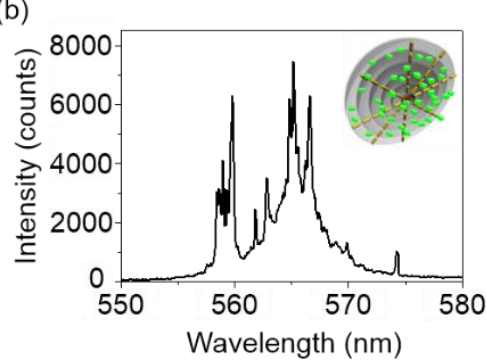

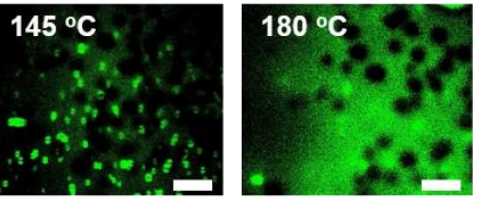

(c)

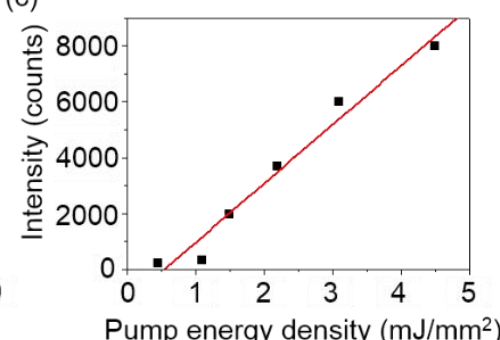

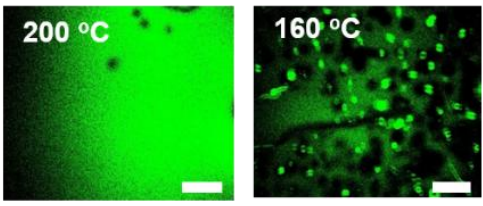

(d)

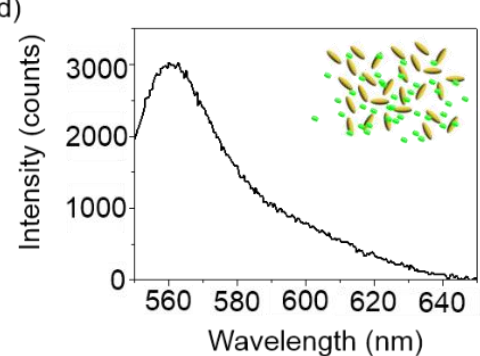

Figure 4. (a) FM images of the sample during the assembly and disassembly of the LC, dyed with R6G, into spheres by thermal phase transition from smectic to isotropic phase. All scale bars are $100 \mu \mathrm{m}$. (b) Lasing spectrum of the dye-doped LC spheres at room temperature. The inset shows a cross sectional sketch of the LC droplet with florescent dyes. (c) Output intensity of the lasing emission as a function of the pumped energy. (d) Fluorescent spectrum in the fully mixed state including R6G dye at $200{ }^{\circ} \mathrm{C}$. 


\section{WILEY-VCH}

The table of contents entry should be 50-60 words long and should be written in the present tense and impersonal style (i.e., avoid we). The text should be different from the abstract text.

Keywords: emulsion, liquid crystal, self-regulation, switchable lasing

Dae Seok Kim, Wonsuk Lee, Teresa Lopez-Leon* and Dong Ki Yoon*

\section{Self-regulated smectic emulsion with switchable lasing application}

ToC figure ((Please choose one size: $55 \mathrm{~mm}$ broad $\times 50 \mathrm{~mm}$ high or $110 \mathrm{~mm}$ broad $\times 20 \mathrm{~mm}$ high. Please do not use any other dimensions))

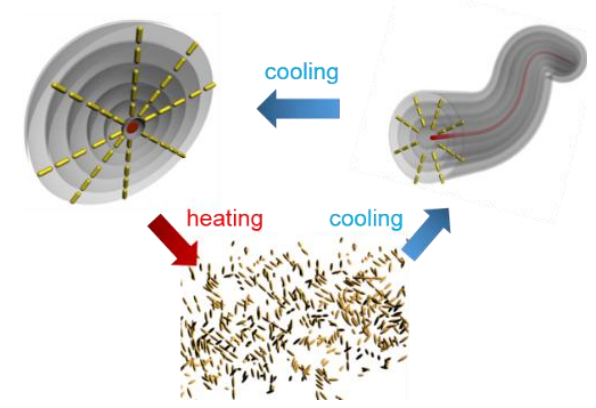

The fluorinated LC molecules spontaneously form monodisperse spherical and myelin-like structures upon cooling from the isotropic phase, while they get dispersed into the continuous medium, i.e. silicon oil, when increasing temperature above the isotropic critical value. The ability of the LC to trap guest molecules, for instance fluorescent dyes, when forming layered LC spheres is exploited to produce a reversible on-off lasing system. 


\section{WILEY-VCH}

Copyright WILEY-VCH Verlag GmbH \& Co. KGaA, 69469 Weinheim, Germany, 2018.

\section{Supporting Information}

Self-regulated smectic emulsion with switchable lasing application

Dae Seok Kim, Wonsuk Lee, Teresa Lopez-Leon* and Dong Ki Yoon*

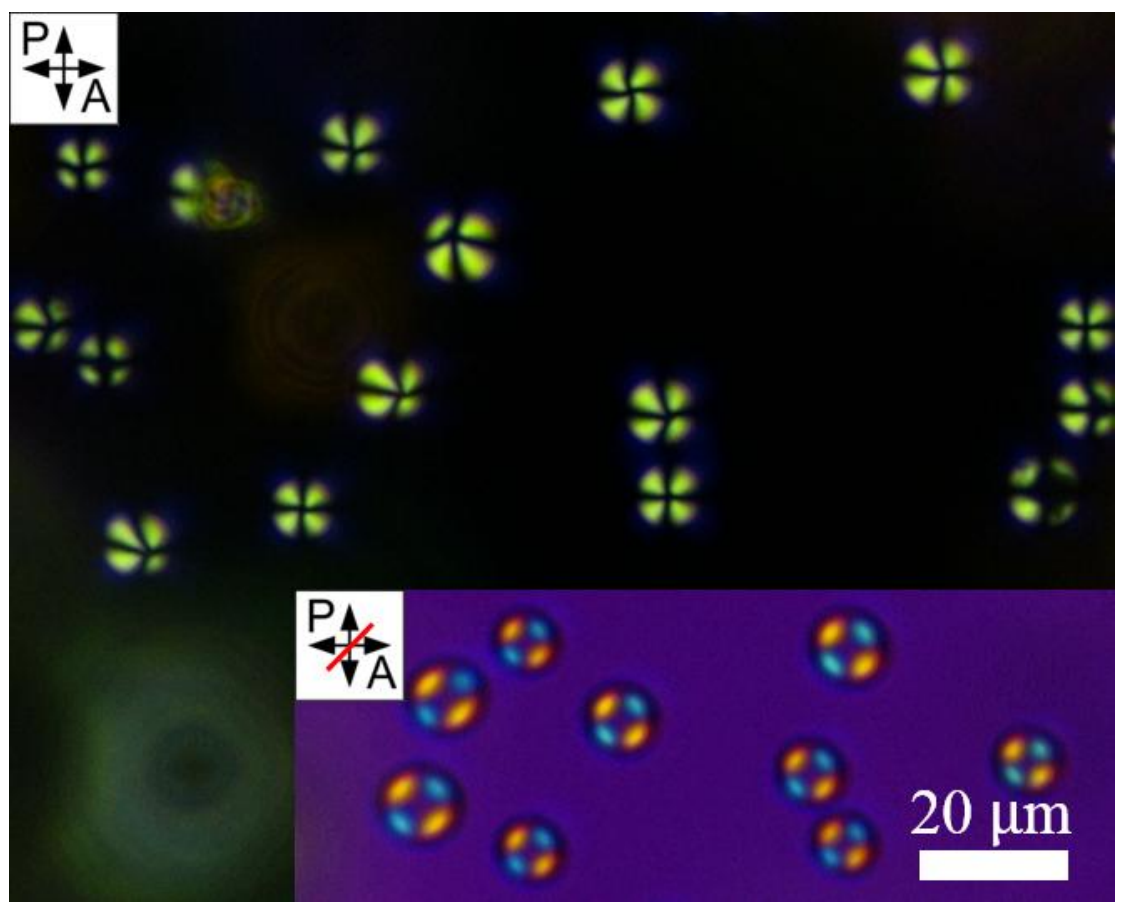

Figure S1. POM image with and without retardation plate (indicated in red) of LC spheres dispersed in silicon oil. 


\section{WILEY-VCH}
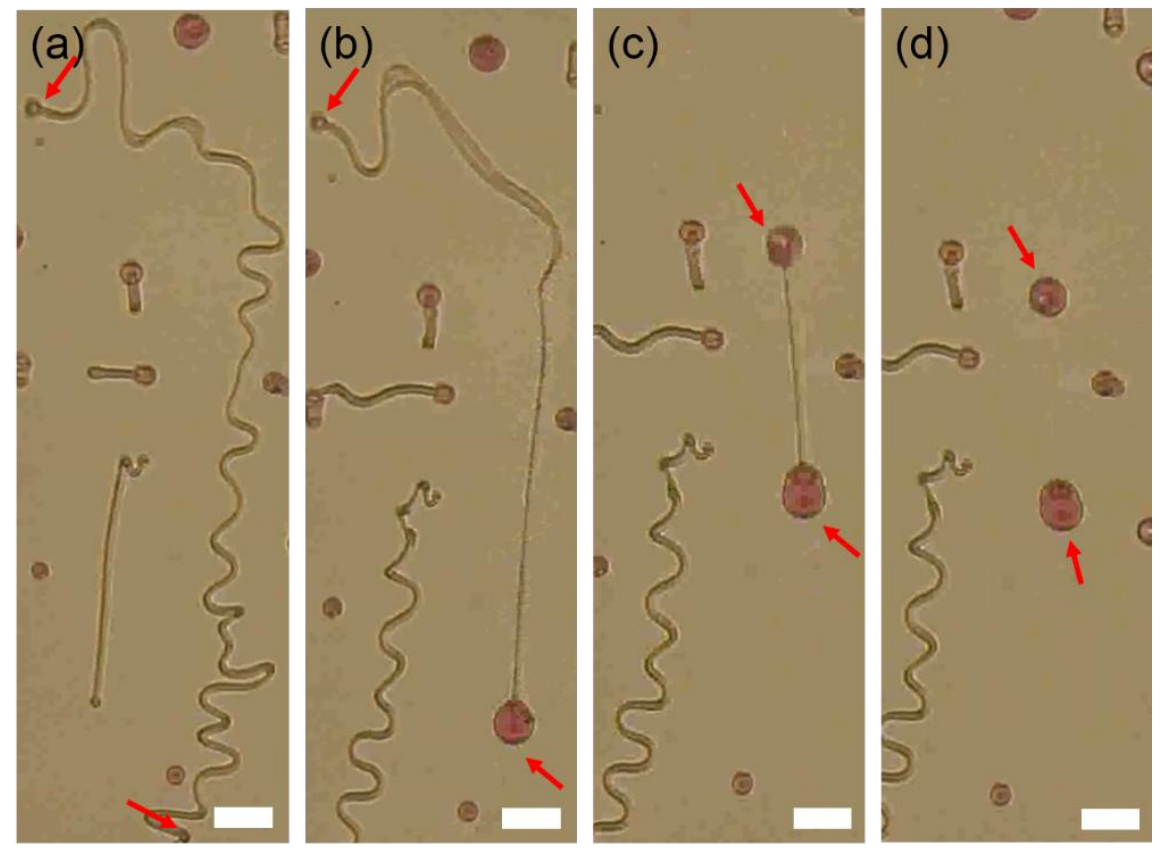

Figure S2. (a-d) Sequential images showing how a long filament splits into two to form two spherical droplets. The images were taken during the cooling of the sample from the isotropic to the smectic transition temperature $(\sim 1950 \mathrm{C})$. Scale bars are $20 \mu \mathrm{m}$. 


\section{WILEY-VCH}
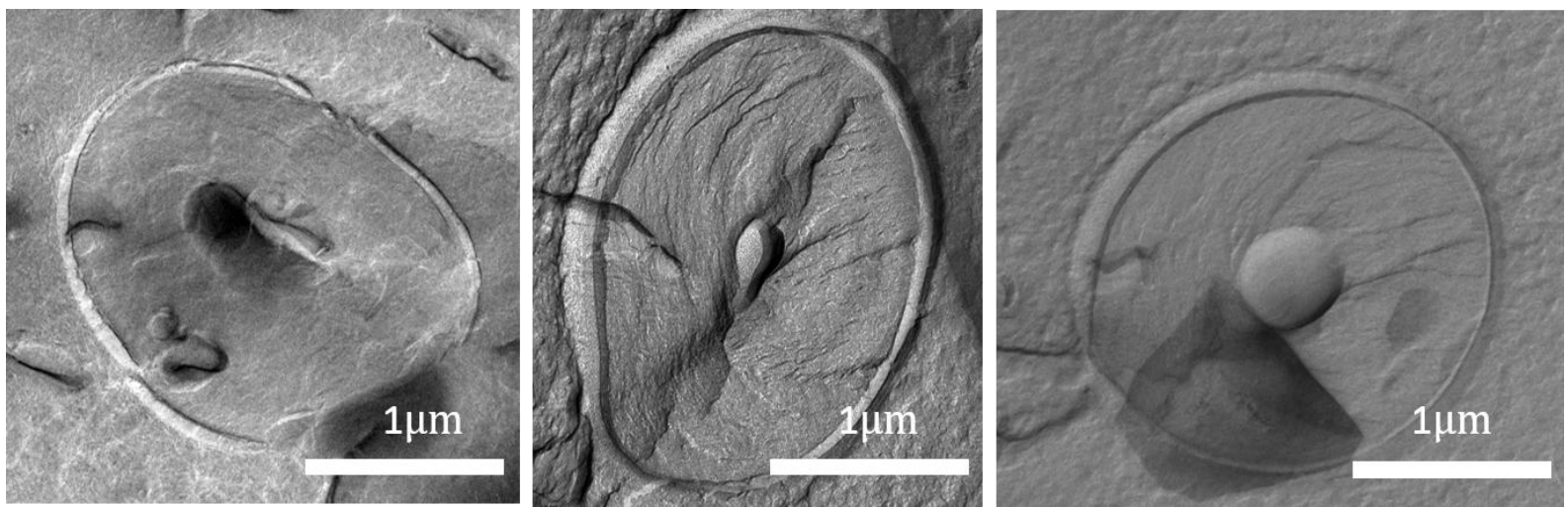

Figure S3. Cross section of a smectic filament showing the smectic layering nucleation

process. The images are obtained from FF-TEM when the sample is quenched rapidly from the isotropic-smectic transition temperature to room temperature. 


\section{WILEY-VCH}
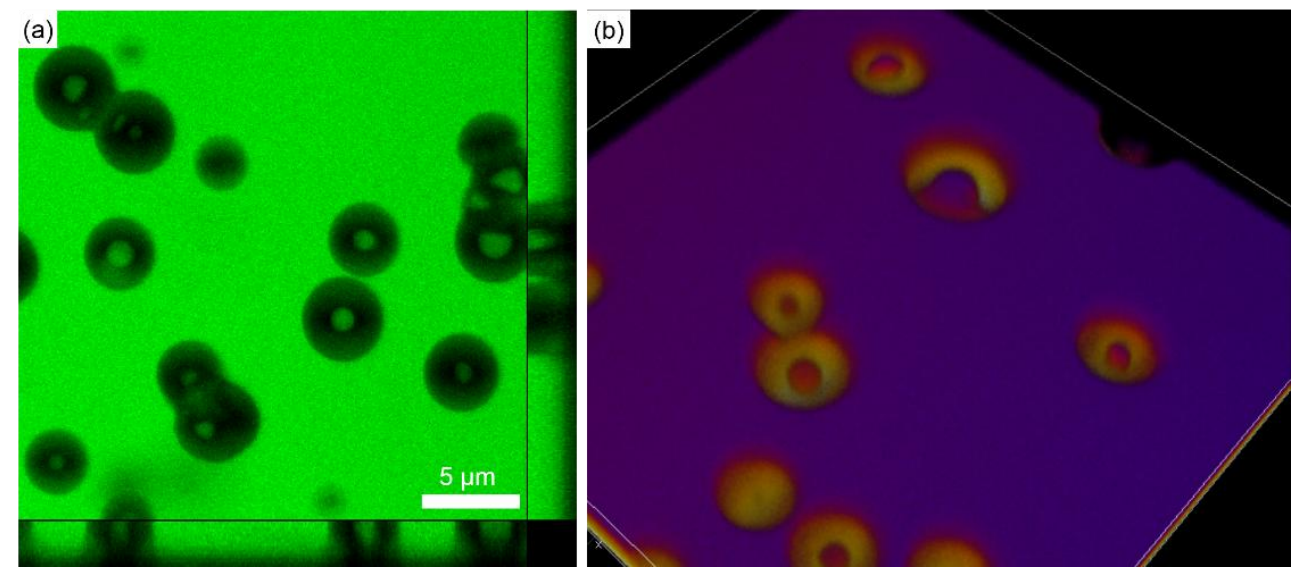

Figure S4. Fluorescent images of smectic droplets produced by quenching a LC mixture that contains silicon oil doped with a fluorescent dye (Coumarin 153) and Y002 from 200oC to room temperature. (a) 2D image (b) 3D image. 


\section{WILEY-VCH}

(a)

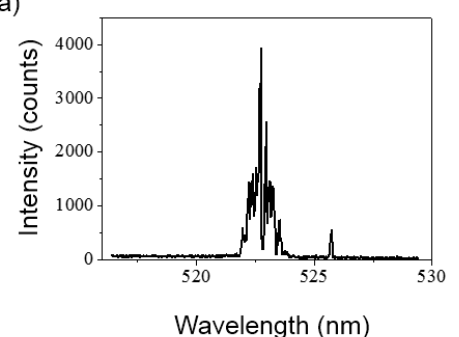

(b)

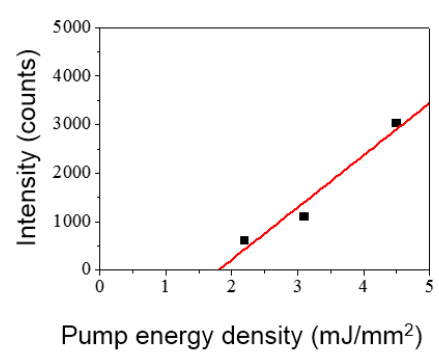

(c)

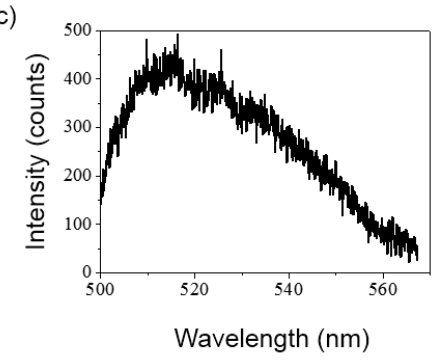

Figure S5. (a) Lasing spectrum in the WGM on the LC spheres at room temperature. (b) Output intensity of the lasing emission as a function of the pumped energy. (c) Fluorescent spectrum in the fully mixed state including the BTBP dye at $200{ }^{\circ} \mathrm{C}$. 


\section{WILEY-VCH}

Transfer to diluted Si Oil
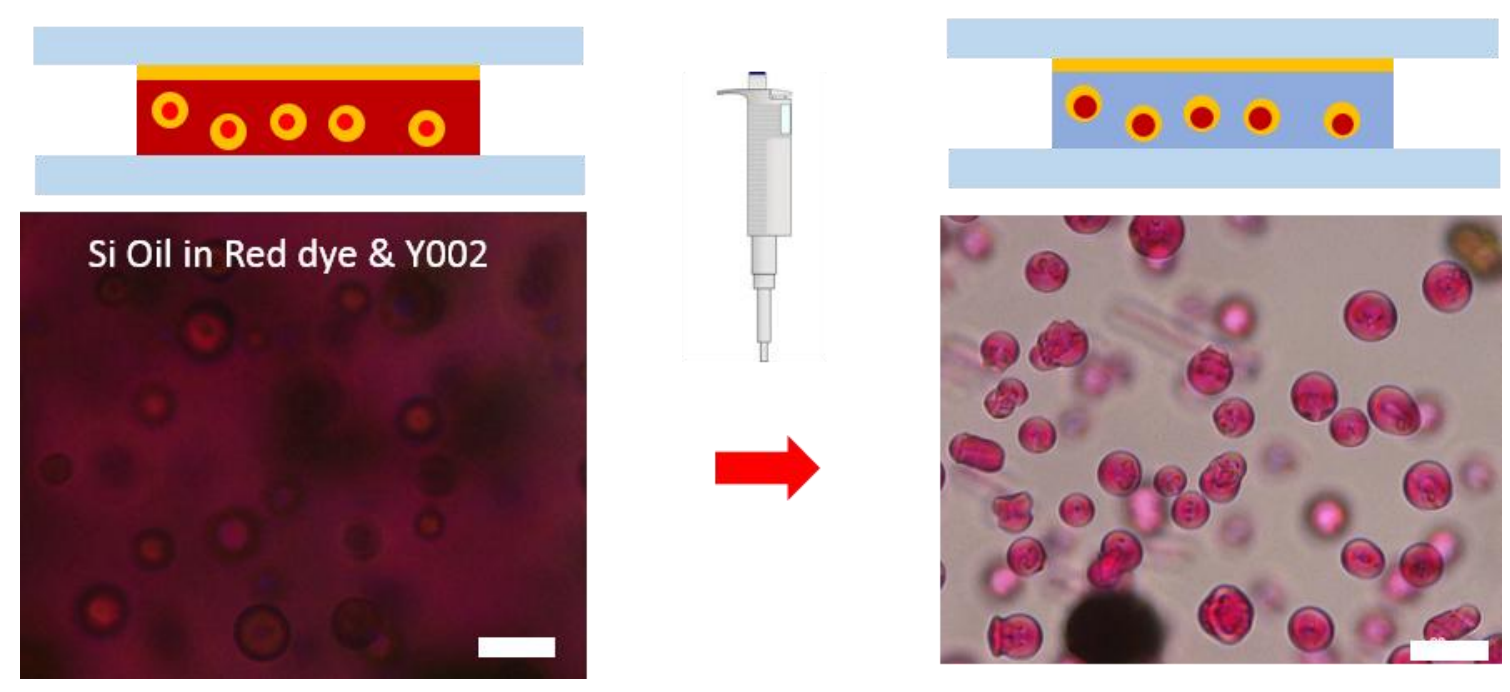

Figure S6. OM images of silicon oil containing LC droplets and red dyes. The sample, initially concentrated in red dyes (left), was diluted by adding pure silicon oil (right). The images show that red dye specifically dopes the LC spheres. 


\section{WILEY-VCH}

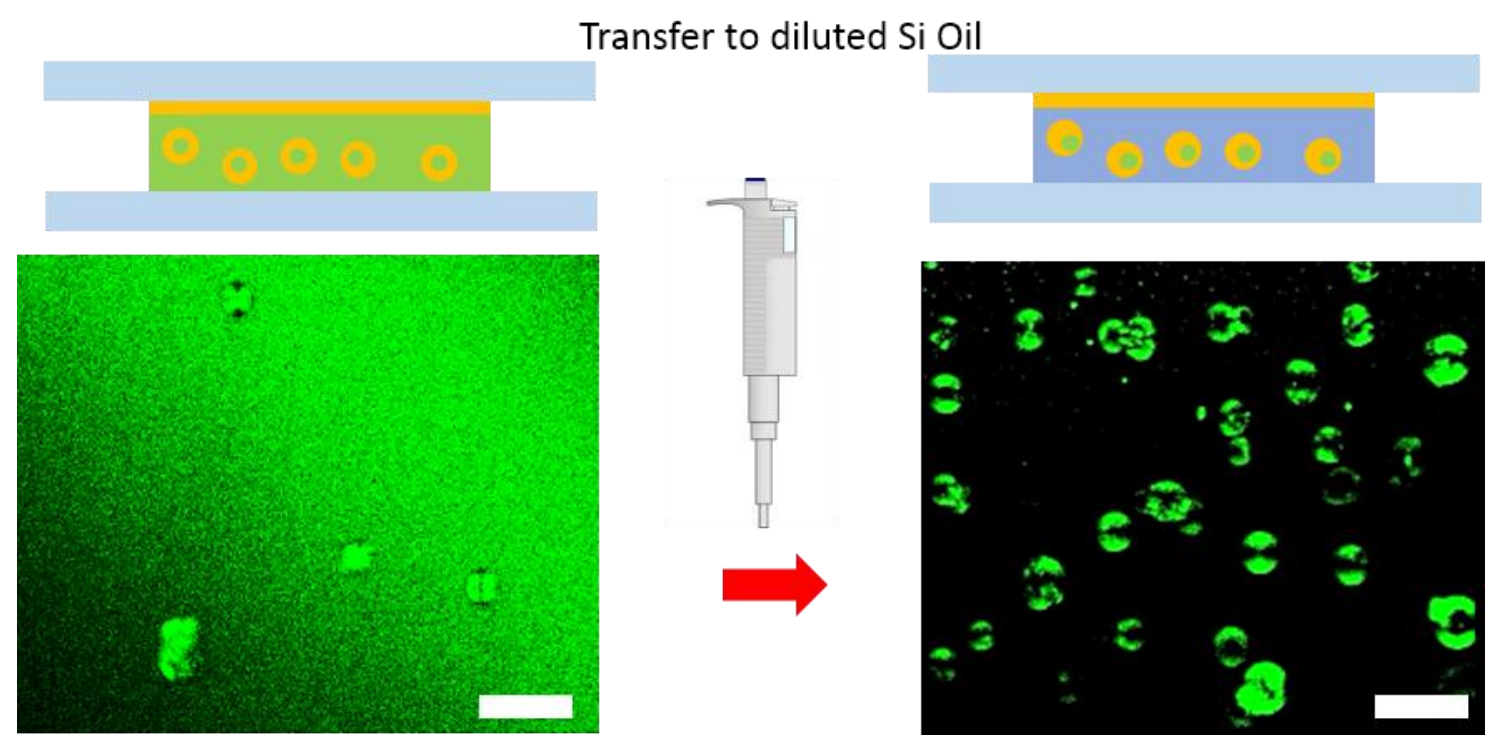

Figure S7. Fluorescent OM images of silicon oil containing LC droplets and BTBP. The sample, initially concentrated in BTBP (left), was diluted by adding pure silicon oil (right). The images show that the fluorescent molecules specifically dope the LC spheres. 